\title{
A Strange Term in the Homogenization of Parabolic Equations with Two Spatial and Two Temporal Scales
}

\author{
L. Flodén, A. Holmbom, and M. Olsson Lindberg \\ Department of Engineering and Sustainable Development, Mid Sweden University, \\ 83125 Östersund, Sweden \\ Correspondence should be addressed to A. Holmbom, anders.holmbom@miun.se
}

Received 23 March 2011; Accepted 28 September 2011

Academic Editor: Bjorn Birnir

Copyright ( 2012 L. Flodén et al. This is an open access article distributed under the Creative Commons Attribution License, which permits unrestricted use, distribution, and reproduction in any medium, provided the original work is properly cited.

\begin{abstract}
We study the homogenization of a parabolic equation with oscillations in both space and time in the coefficient $a\left(x / \varepsilon, t / \varepsilon^{2}\right)$ in the elliptic part and spatial oscillations in the coefficient $\rho(x / \varepsilon)$ that is multiplied with the time derivative $\partial_{t} u^{\varepsilon}$. We obtain a strange term in the local problem. This phenomenon appears as a consequence of the combination of the spatial oscillation in $\rho(x / \varepsilon)$ and the temporal oscillation in $a\left(x / \varepsilon, t / \varepsilon^{2}\right)$ and disappears if either of these oscillations is removed.
\end{abstract}

\section{Introduction}

We study the homogenization of

$$
\begin{gathered}
\rho\left(\frac{x}{\varepsilon}\right) \partial_{t} u^{\varepsilon}(x, t)-\nabla \cdot\left(a\left(\frac{x}{\varepsilon}, \frac{t}{\varepsilon^{2}}\right) \nabla u^{\varepsilon}(x, t)\right)=f(x, t) \quad \text { in } \Omega \times(0, T), \\
u^{\varepsilon}(x, 0)=g(x) \quad \text { in } \Omega, \\
u^{\varepsilon}(x, t)=0 \quad \text { on } \partial \Omega \times(0, T),
\end{gathered}
$$

which contains oscillations in both space and time in the coefficient $a\left(x / \varepsilon, t / \varepsilon^{2}\right)$ in the elliptic part and spatial oscillations in the coefficient $\rho(x / \varepsilon)$ that is multiplied with the time derivative $\partial_{t} u^{\varepsilon}$. The technique is an adaption of two-scale convergence to parabolic homogenization. To deal with the oscillations of $\rho(x / \varepsilon)$, we need to make a special choice of test functions for our approach to apply, which is the reason why an additional term is obtained in the local problem. This phenomenon appears as a consequence of the combination 
of the spatial oscillation in $\rho(x / \varepsilon)$ and the temporal oscillation in $a\left(x / \varepsilon, t / \varepsilon^{2}\right)$ and disappears if either of these oscillations is removed. Understanding (1.1) in terms of physics, the coefficient $\rho(x / \varepsilon)$ means that the density and the heat capacity may follow a pattern of spatial heterogeneity similar to the thermal conductivity. It is worth noting that the strange term in the local problem appears with a coefficient $\rho(x / \varepsilon)$ with spatial oscillations with the same frequency as the heat conductivity coefficient but without the corresponding temporal oscillations. To the authors' knowledge the physical interpretation of this phenomenon remains to be understood.

A related problem is studied by Nandakumaran and Rajesh in [1], with the temporal oscillations of the same frequency as the spatial ones and hence the resonance phenomenon in the local problem that we obtain for (1.1) does not appear; see also Remarks 3.3 and 3.4. They investigate

$$
\partial_{t} \rho\left(\frac{x}{\varepsilon}, u^{\varepsilon}\right)-\nabla \cdot a\left(\frac{x}{\varepsilon}, \frac{t}{\varepsilon}, u^{\varepsilon}, \nabla u^{\varepsilon}\right)=f(x, t) \quad \text { in } \Omega \times(0, T)
$$

with mixed boundary conditions under certain continuity and monotonicity assumptions on $\rho$ and $a$. There will turn out to be a significant difference between the treatment of the cases where the speed of the temporal oscillations is governed by $\varepsilon$, as in (1.2), and $\varepsilon^{2}$, which is considered in the main result of this paper. Simpler linear problems without temporal oscillations are found in, for example, $[2,3]$.

\section{Two-Scale Convergence}

Our main tools are some versions of two-scale convergence. Two-scale convergence was first introduced by Nguetseng in [4]. The definition below was established by Allaire in [5] and has become the standard way to define two-scale convergence. It is a slight modification of the original definition in [4].

Notation 1. $F_{\#}(Y)$ means the space of all functions in $F_{\text {loc }}\left(\mathbb{R}^{N}\right)$ that are $Y$-periodic repetitions of some function in $F(Y)$. $\Omega$ is a bounded open set in $\mathbb{R}^{N}$ with a smooth boundary and $\Omega_{T}=$ $\Omega \times(0, T)$.

Definition 2.1. One says that a sequence $\left\{u^{\varepsilon}\right\}$ in $L^{2}(\Omega)$ two-scale converges to $u_{0} \in L^{2}(\Omega \times Y)$ if

$$
\int_{\Omega} u^{\varepsilon}(x) v\left(x, \frac{x}{\varepsilon}\right) d x \rightarrow \int_{\Omega} \int_{Y} u_{0}(x, y) v(x, y) d y d x
$$

for any $v \in L^{2}\left(\Omega ; C_{\#}(Y)\right)$ when $\varepsilon \rightarrow 0$. One writes $u^{\varepsilon} \stackrel{2}{\rightarrow} u_{0}$.

Translating to the appropriate evolution setting we introduce the next variant. 
Definition 2.2. One says that a sequence $\left\{\mathcal{u}^{\varepsilon}\right\}$ in $L^{2}\left(\Omega_{T}\right)(2,2)$-scale converges to $u_{0} \in L^{2}\left(\Omega_{T} \times\right.$ $Y \times(0,1))$ if

$$
\int_{\Omega_{T}} u^{\varepsilon}(x, t) v\left(x, t, \frac{x}{\varepsilon}, \frac{t}{\varepsilon^{2}}\right) d x d t \longrightarrow \int_{\Omega_{T}} \int_{0}^{1} \int_{Y} u_{0}(x, t, y, s) v(x, t, y, s) d y d s d x d t
$$

for any $v \in L^{2}\left(\Omega_{T} ; C_{\#}(Y \times(0,1))\right)$ when $\varepsilon \rightarrow 0$. One writes

$$
u^{\varepsilon}(x, t) \stackrel{2,2}{\rightarrow} u_{0}(x, t, y, s) .
$$

The somewhat weaker type of convergence defined next is an essential tool in the homogenization of (1.1) and under certain assumptions works without the requirement on boundedness in $L^{2}$ which is necessary to obtain convergence up to a subsequence in usual two-scale convergence, see [6].

Definition 2.3. One says that a sequence $\left\{w^{\varepsilon}\right\}$ in $L^{1}\left(\Omega_{T}\right)(2,2)$-scale converges very weakly to $w_{0} \in L^{1}\left(\Omega_{T} \times Y \times(0,1)\right)$ if

$$
\begin{aligned}
\int_{\Omega_{T}} w^{\varepsilon}(x, t) v_{1}(x) v_{2}\left(\frac{x}{\varepsilon}\right) c_{1}(t) c_{2}\left(\frac{t}{\varepsilon^{2}}\right) d x d t \\
\quad \longrightarrow \int_{\Omega_{T}} \int_{0}^{1} \int_{Y} w_{0}(x, t, y, s) v_{1}(x) v_{2}(y) c_{1}(t) c_{2}(s) d y d s d x d t
\end{aligned}
$$

for any $v_{1} \in D(\Omega), v_{2} \in C_{\#}^{\infty}(Y) / \mathbb{R}, c_{1} \in D(0, T)$, and $c_{2} \in C_{\#}^{\infty}(0,1)$ when $\varepsilon \rightarrow 0$. One writes

$$
u^{\varepsilon}(x, t) \frac{2,2}{v w} u_{0}(x, t, y, s) .
$$

Let $W_{2}^{1}\left(0, T ; H_{0}^{1}(\Omega), L^{2}(\Omega)\right)$ be the space of all functions in $L^{2}\left(0, T ; H_{0}^{1}(\Omega)\right)$ such that the time derivative belongs to $L^{2}\left(0, T ; H^{-1}(\Omega)\right)$; see, for example, [7, Chapter 23]. For $\left\{u^{\varepsilon}\right\}$ bounded in $W_{2}^{1}\left(0, T ; H_{0}^{1}(\Omega), L^{2}(\Omega)\right)$ we also have a characterization of the $(2,2)$-scale limit for the gradients $\nabla u^{\varepsilon}$ and the corresponding very weak limit for $\left\{u^{\varepsilon} / \varepsilon\right\}$.

Theorem 2.4. Let $\left\{u^{\varepsilon}\right\}$ be a bounded sequence in $W_{2}^{1}\left(0, T ; H_{0}^{1}(\Omega), L^{2}(\Omega)\right)$. Then, there exists a subsequence such that

$$
\begin{gathered}
u^{\varepsilon}(x, t) \longrightarrow u(x, t) \quad \text { in } L^{2}\left(\Omega_{T}\right), \\
u^{\varepsilon}(x, t) \rightarrow u(x, t) \quad \text { in } L^{2}\left(0, T ; H_{0}^{1}(\Omega)\right), \\
\nabla u^{\varepsilon}(x, t) \stackrel{2,2}{\longrightarrow} \nabla u(x, t)+\nabla_{y} u_{1}(x, t, y, s),
\end{gathered}
$$

where $u \in W_{2}^{1}\left(0, T ; H_{0}^{1}(\Omega), L^{2}(\Omega)\right)$ and $u_{1} \in L^{2}\left(\Omega_{T} \times(0,1) ; H_{\#}^{1}(Y) / \mathbb{R}\right)$. Moreover

$$
\frac{u^{\varepsilon}(x, t)}{\varepsilon} \frac{2,2}{v w} u_{1}(x, t, y, s)
$$


Proof. The results in (2.7) and (2.8) can be seen as the period special case for the corresponding results in terms of $\Sigma$-convergence in [8]; see, for example, Defintion 3.1, Lemma 3.4 and Section 4.2 in [8]. We can also obtain (2.7) by a slight modification of the standard proof for bounded sequences in $H^{1}(\Omega)$ if we observe (2.6), that is, that any bounded sequence in $W_{2}^{1}\left(0, T ; H_{0}^{1}(\Omega), L^{2}(\Omega)\right)$ contains a subsequence that converges strongly in $L^{2}\left(\Omega_{T}\right)$; see, for example, [9]. In the same way (2.8) can be concluded from [6, Theorem 4].

Remark 2.5. Limits of the type in (2.8) appear in the proof of the homogenization result for (1.1) in Section 3. The important point here is to find a limit for a sequence $\left\{u^{\varepsilon} / \varepsilon\right\}$, where the denominator $\varepsilon$ passes to zero, while the numerator $u^{\varepsilon}$ does not. The reason why we assume that $\int_{Y} v_{2}(y) d y=0$ in Definition 2.3 is that $v_{2}$ has to be generated in a certain manner for the proof of (2.8) to work. This is not so with, for example, $c_{2}$; see, for example, $[6,8,9]$.

Remark 2.6. The results in Theorem 2.4 can also be obtained under the assumption that $\left\{u^{\varepsilon}\right\}$ apart from being a sequence of solutions to (1.1) and hence bounded in $L^{2}\left(0, T ; H_{0}^{1}(\Omega)\right)$ is also bounded in $L^{\infty}\left(\Omega_{T}\right)$. These conditions together imply that $\left\{u^{\varepsilon}\right\}$ converges strongly in $L^{2}\left(\Omega_{T}\right)$ up to a subsequence and hence the boundedness of $\left\{u^{\varepsilon}\right\}$ in $L^{\infty}\left(\Omega_{T}\right)$ replaces the boundedness of $\left\{\partial_{t} u^{\varepsilon}\right\}$ in $L^{2}\left(0, T ; H^{-1}(\Omega)\right)$; see Lemma 3.3 and (4.1) in [1]. The proof is then possible to perform in the same way as for $\left\{u^{\varepsilon}\right\}$ bounded in $W_{2}^{1}\left(0, T ; H_{0}^{1}(\Omega), L^{2}(\Omega)\right)$. The only difference is that $u$ will belong to $L^{2}\left(0, T ; H_{0}^{1}(\Omega)\right)$ instead of the space $W_{2}^{1}\left(0, T ; H_{0}^{1}(\Omega), L^{2}(\Omega)\right)$.

\section{Homogenization}

We develop a homogenization procedure for (1.1) and obtain the result in the theorem below. Omitting the rapid temporal oscillations, that is, replacing $a\left(x / \varepsilon, t / \varepsilon^{2}\right)$ with $a(x / \varepsilon)$, there are no important consequences of the appearance of $\rho(x / \varepsilon)$ and the local problem would be the same as for $\rho=1$. With the temporal oscillations the situation is, however, sometimes different from what it should have been with, for example, $\rho=1$. We need to apply (2.8) to find the local problem but encounter a difficulty in the sense that $\rho v$ does not in general have average zero over $Y$ for $v \in C_{\#}^{\infty}(Y)$ or even $v \in C_{\#}^{\infty}(Y) / \mathbb{R}$. This necessitates a construction of special test functions to be used in the weak form (3.6) of (1.1) in the proof of Theorem 3.1. We assume that $\rho \in C_{\#}^{\infty}(Y), \rho \geq C>0, f \in L^{2}\left(\Omega_{T}\right)$, and

$$
a(y, s) c \cdot c \geq A|c|^{2}, \quad A>0
$$

where $a \in L_{\#}^{\infty}(Y \times(0,1))^{N \times N}$. It can be proven along the lines of the corresponding proof in [7, Section 23.9] that $\left\{u^{\varepsilon}\right\}$ is bounded in the space $L^{2}\left(0, T ; H_{0}^{1}(\Omega)\right)$; see also [2]. For, for example, $\rho=1$ it also holds that $\left\{\partial_{t} \mathcal{u}^{\varepsilon}\right\}$ is bounded in $L^{2}\left(0, T ; H^{-1}(\Omega)\right)$ and hence $\left\{u^{\varepsilon}\right\}$ is bounded in the stronger space $W_{2}^{1}\left(0, T ; H_{0}^{1}(\Omega), L^{2}(\Omega)\right)$. Here, we instead make the physically quite natural assumption that $\left\{u^{\varepsilon}\right\}$ is bounded in $L^{\infty}\left(\Omega_{T}\right)$; see [1] and the references therein.

Theorem 3.1. Let $\left\{u^{\varepsilon}\right\}$ be a sequence of solutions to (1.1), where $\varepsilon \rightarrow 0$, and assume that $\left\{u^{\varepsilon}\right\}$ is bounded in $L^{\infty}\left(\Omega_{T}\right)$. Then,

$$
u^{\varepsilon} \rightarrow u \quad \text { in } L^{2}\left(0, T ; H_{0}^{1}(\Omega)\right)
$$


where $u$ is the solution to

$$
\begin{gathered}
\int_{Y} \rho(y) d y \partial_{t} u(x, t)-\nabla \cdot(b \nabla u(x, t))=f(x, t) \quad \text { in } \Omega_{T}, \\
u(x, 0)=g(x) \quad \text { in } \Omega, \\
u(x, t)=0 \quad \text { on } \partial \Omega \times(0, T),
\end{gathered}
$$

with

$$
b \nabla u(x, t)=\int_{0}^{1} \int_{Y} a(y, s)\left(\nabla u(x, t)+\nabla_{y} u_{1}(x, t, y, s)\right) d y d s
$$

where $u_{1} \in L^{2}\left(\Omega_{T} \times(0,1) ; H_{\#}^{1}(Y) / \mathbb{R}\right)$ solves

$$
\begin{aligned}
& \rho(y) \partial_{s} u_{1}(x, t, y, s)-\nabla_{y} \cdot\left(a(y, s)\left(\nabla u(x, t)+\nabla_{y} u_{1}(x, t, y, s)\right)\right) \\
& =\rho(y)\left(\int_{Y} a(y, s)\left(\nabla u(x, t)+\nabla_{y} u_{1}(x, t, y, s)\right) \cdot \nabla_{y}\left(\frac{1}{\rho(y)}\right) d y\right) .
\end{aligned}
$$

Remark 3.2. For $\rho=1$, the right-hand side of (3.5) is zero and hence the strange term in the local problem disappears. An interesting question is to investigate if there are ways to make the strange term disappear and obtain a more conventional local problem also when $\rho$ is oscillating. This would simplify the use of standard software for the solution of the local problem. See Remark 3.3, where this is discussed for the case where the temporal oscillations are of the same frequency as the spatial ones, and Remark 3.4.

Proof. Let us study the weak form

$$
\begin{gathered}
\int_{\Omega_{T}}-\rho\left(\frac{x}{\varepsilon}\right) u^{\varepsilon}(x, t) v(x) \partial_{t} c(t)+a\left(\frac{x}{\varepsilon}, \frac{t}{\varepsilon^{2}}\right) \nabla u^{\varepsilon}(x, t) \cdot \nabla v(x) c(t) d x d t \\
=\int_{\Omega_{T}} f(x, t) v(x) c(t) d x d t
\end{gathered}
$$

$v \in H_{0}^{1}(\Omega), c \in D(0, T)$ of (1.1). We apply (2.6) and (2.7), pass to the limit and arrive, up to a subsequence, at the homogenized problem

$$
\begin{gathered}
\int_{\Omega_{T}} \int_{0}^{1} \int_{Y}-\rho(\mathrm{y}) u(x, t) v(x) \partial_{t} c(t)+a(y, s)\left(\nabla u(x, t)+\nabla_{y} u_{1}(x, t, y, s)\right) \cdot \nabla v(x) c(t) d y d s d x d t \\
=\int_{\Omega_{T}} f(x, t) v(x) c(t) d x d t .
\end{gathered}
$$


To find a local problem we choose

$$
v(x)=\varepsilon v_{1}(x) \widetilde{v}\left(\frac{x}{\varepsilon}\right)
$$

in (3.6), where $v_{1} \in D(\Omega)$,

$$
\begin{gathered}
\tilde{v}(y)=v_{2}(y)-\frac{C}{\rho(y)}, \quad v_{2} \in C_{\#}^{\infty}(Y), \\
C=\int_{Y} \rho(y) v_{2}(y) d y .
\end{gathered}
$$

Hence, we have

$$
\int_{Y} \rho(y) \tilde{v}(y) d y=0
$$

Further, we let

$$
c(t)=c_{1}(t) c_{2}\left(\frac{t}{\varepsilon^{2}}\right), \quad c_{1} \in D(0, T), c_{2} \in C_{\#}^{\infty}(0,1)
$$

and arrive at

$$
\begin{aligned}
& \int_{\Omega_{T}}-\rho\left(\frac{x}{\varepsilon}\right) u^{\varepsilon}(x, t) v_{1}(x) \tilde{v}\left(\frac{x}{\varepsilon}\right)\left(\varepsilon \partial_{t} c_{1}(t) c_{2}\left(\frac{t}{\varepsilon^{2}}\right)+\varepsilon^{-1} c_{1}(t) \partial_{s} c_{2}\left(\frac{t}{\varepsilon^{2}}\right)\right) \\
& \quad+a\left(\frac{x}{\varepsilon}, \frac{t}{\varepsilon^{2}}\right) \nabla u^{\varepsilon}(x, t) \cdot\left(\varepsilon \nabla v_{1}(x) \tilde{v}\left(\frac{x}{\varepsilon}\right)+v_{1}(x) \nabla_{y} \tilde{v}\left(\frac{x}{\varepsilon}\right)\right) c_{1}(t) c_{2}\left(\frac{t}{\varepsilon^{2}}\right) d x d t \\
& =\int_{\Omega_{T}} f(x, t) \varepsilon v_{1}(x) \tilde{v}\left(\frac{x}{\varepsilon}\right) c_{1}(t) c_{2}\left(\frac{t}{\varepsilon^{2}}\right) d x d t .
\end{aligned}
$$

The choice of $\widetilde{v}$ is motivated by the requirement that we should have (3.11) for (2.8) to be applicable. We let $\varepsilon \rightarrow 0$ in (3.13) and apply (2.7) and (2.8) to

$$
\begin{gathered}
\int_{\Omega_{T}} a\left(\frac{x}{\varepsilon}, \frac{t}{\varepsilon^{2}}\right) \nabla u^{\varepsilon}(x, t) \cdot v_{1}(x) \nabla_{y} \tilde{v}\left(\frac{x}{\varepsilon}\right) c_{1}(t) c_{2}\left(\frac{t}{\varepsilon^{2}}\right) d x d t \\
\int_{\Omega_{T}}-\rho\left(\frac{x}{\varepsilon}\right) u^{\varepsilon}(x, t) v_{1}(x) \tilde{v}\left(\frac{x}{\varepsilon}\right) \varepsilon^{-1} c_{1}(t) \partial_{s} c_{2}\left(\frac{t}{\varepsilon^{2}}\right) d x d t
\end{gathered}
$$


Journal of Function Spaces and Applications

respectively. Noting that the rest of terms in (3.13) vanish, we obtain, up to a subsequence,

$$
\begin{gathered}
\int_{\Omega_{T}} \int_{0}^{1} \int_{Y}-\rho(y) u_{1}(x, t, y, s) v_{1}(x) \tilde{v}(y) c_{1}(t) \partial_{s} c_{2}(s)+a(y, s)\left(\nabla u(x, t)+\nabla_{y} u_{1}(x, t, y, s)\right) \\
\cdot v_{1}(x) \nabla_{y} \tilde{v}(y) c_{1}(t) c_{2}(s) d y d s d x d t=0 .
\end{gathered}
$$

Hence, observing that

$$
\int_{Y}-\rho(y) u_{1}(x, t, y, s)\left(-\frac{C}{\rho(y)}\right) d y=\int_{Y} C u_{1}(x, t, y, s) d y=0
$$

and recalling (3.9), we arrive at

$$
\begin{gathered}
\int_{\Omega_{T}} \int_{0}^{1} \int_{Y}-\rho(y) u_{1}(x, t, y, s) v_{1}(x) v_{2}(y) c_{1}(t) \partial_{s} c_{2}(s)+a(y, s)\left(\nabla u(x, t)+\nabla_{y} u_{1}(x, t, y, s)\right) \\
\cdot v_{1}(x) \nabla_{y}\left(v_{2}(y)-\frac{C}{\rho(y)}\right) c_{1}(t) c_{2}(s) d y d s d x d t=0 .
\end{gathered}
$$

We write

$$
\begin{aligned}
& \int_{\Omega_{T}} \int_{0}^{1} \int_{Y}-\rho(y) u_{1}(x, t, y, s) v_{1}(x) v_{2}(y) c_{1}(t) \partial_{s} c_{2}(s) \\
& +a(y, s)\left(\nabla u(x, t)+\nabla_{y} u_{1}(x, t, y, s)\right) \cdot v_{1}(x) \nabla_{y} v_{2}(y) c_{1}(t) c_{2}(s) d y d s d x d t \\
& -\int_{\Omega_{T}} \int_{0}^{1}\left(\int_{Y} a(y, s)\left(\nabla u(x, t)+\nabla_{y} u_{1}(x, t, y, s)\right) \cdot v_{1}(x) \nabla_{y}\left(\frac{C}{\rho(y)}\right) d y\right) \cdot c_{1}(t) c_{2}(s) d s d x d t
\end{aligned}
$$

Applying repeatedly the variational lemma, we find that

$$
\begin{aligned}
& \int_{0}^{1} \int_{Y}-\rho(y) u_{1}(x, t, y, s) v_{2}(y) \partial_{s} c_{2}(s) \\
& +a(y, s)\left(\nabla u(x, t)+\nabla_{y} u_{1}(x, t, y, s)\right) \cdot \nabla_{y} v_{2}(y) c_{2}(s) d y d s \\
& =\int_{0}^{1}\left(\int_{Y} a(y, s)\left(\nabla u(x, t)+\nabla_{y} u_{1}(x, t, y, s)\right) \cdot \nabla_{y}\left(\frac{C}{\rho(y)}\right) d y\right) c_{2}(s) d s
\end{aligned}
$$


and, according to the definition (3.10) of $C$,

$$
\begin{aligned}
& \int_{0}^{1} \int_{Y}-\rho(y) u_{1}(x, t, y, s) v_{2}(y) \partial_{s} c_{2}(s) \\
& \quad+a(y, s)\left(\nabla u(x, t)+\nabla_{y} u_{1}(x, t, y, s)\right) \cdot \nabla_{y} v_{2}(y) c_{2}(s) d y d s \\
& =\int_{0}^{1} \int_{Y} \rho(y)\left(\int_{Y} a(y, s)\left(\nabla u(x, t)+\nabla_{y} u_{1}(x, t, y, s)\right) \cdot \nabla_{y}\left(\frac{1}{\rho(y)}\right) d y\right) \cdot v_{2}(y) c_{2}(s) d y d s
\end{aligned}
$$

which is the weak form of (3.5).

Remark 3.3. We also briefly comment on case (1.2) originally studied in [1]. Restricting (1.2) to the linear setting studied in this paper means that we obtain

$$
\rho\left(\frac{x}{\varepsilon}\right) \partial_{t} u^{\varepsilon}(x, t)-\nabla \cdot\left(a\left(\frac{x}{\varepsilon}, \frac{t}{\varepsilon}\right) \nabla u^{\varepsilon}(x, t)\right)=f(x, t) \quad \text { in } \Omega_{T} .
$$

Introducing test functions corresponding to those used to find the local problem (3.5) in the weak form of (3.21), we arrive at

$$
\begin{aligned}
& \int_{\Omega_{T}}-\rho\left(\frac{x}{\varepsilon}\right) u^{\varepsilon}(x, t) v(x) \widetilde{v}\left(\frac{x}{\varepsilon}\right)\left(\varepsilon \partial_{t} c_{1}(t) c_{2}\left(\frac{t}{\varepsilon}\right)+c_{1}(t) \partial_{s} c_{2}\left(\frac{t}{\varepsilon}\right)\right) \\
& \quad+a\left(\frac{x}{\varepsilon}, \frac{t}{\varepsilon}\right) \nabla u^{\varepsilon}(x, t) \cdot\left(\varepsilon \nabla v(x) \tilde{v}\left(\frac{x}{\varepsilon}\right)+v_{1}(x) \nabla_{y} \tilde{v}\left(\frac{x}{\varepsilon}\right)\right) c_{1}(t) c_{2}\left(\frac{t}{\varepsilon}\right) d x d t \\
& =\int_{\Omega_{T}} f(x, t) \varepsilon v(x) \tilde{v}\left(\frac{x}{\varepsilon}\right) c_{1}(t) c_{2}\left(\frac{t}{\varepsilon}\right) d x d t .
\end{aligned}
$$

Letting $\varepsilon$ go to zero, we find, following the same procedure as in the proof of Theorem 3.1, that

$$
\begin{aligned}
-\nabla_{y} \cdot & \left(a(y, s)\left(\nabla u(x, t)+\nabla_{y} u_{1}(x, t, y, s)\right)\right) \\
= & \rho(y)\left(\int_{Y} a(y, s)\left(\nabla u(x, t)+\nabla_{y} u_{1}(x, t, y, s)\right) \cdot \nabla_{y}\left(\frac{1}{\rho(y)}\right) d y\right),
\end{aligned}
$$

and hence it seems like a strange term has appeared also in the local problem for the homogenization of (3.21). However, we do not need very weak two-scale convergence to pass to the limit in (3.22), and hence we can replace $\tilde{v}$ with any $v_{2} \in C_{\#}^{\infty}(Y)$ and obtain the more conventional local problem

$$
-\nabla_{y} \cdot\left(a(y, s)\left(\nabla u(x, t)+\nabla_{y} u_{1}(x, t, y, s)\right)\right)=0
$$

without any strange term. Observing that $1 / \rho \in C_{\#}^{\infty}(Y)$ and hence is an admissible choice of the test function $v_{2}$ in the weak form of (3.24), this means that the right-hand side in (3.23) is zero and hence (3.23) reduces to (3.24). 
Remark 3.4. The question of obtaining a cancellation of the strange term for the homogenization of (1.1) similar to what we saw in Remark 3.3 is delicate. For $\left\{\partial_{t} \mathcal{u}^{\varepsilon}\right\}$ bounded in $L^{2}\left(\Omega_{T}\right)$, a such cancellation appears but under the present conditions of boundedness of $\left\{u^{\varepsilon}\right\}$ in $L^{2}\left(0, T ; H_{0}^{1}(\Omega)\right)$ and $L^{\infty}\left(\Omega_{T}\right)$ and strong convergence in $L^{2}\left(\Omega_{T}\right)$ there are counterexamples. Hence, this far, we have only found ways to neutralize the strange term in (3.5) by means of nonstandard boundedness assumptions for (1.1). Forthcoming studies will address these questions in more detail.

\section{References}

[1] A. K. Nandakumaran and M. Rajesh, "Homogenization of a nonlinear degenerate parabolic differential equation," Electronic Journal of Differential Equations, vol. 2001, no. 17, pp. 1-19, 2001.

[2] L. Persson, L. E. Persson, N. Svanstedt, and J. Wyller, The Homogenization Method, Studentlitteratur, Lund, Chartwell-Bratt Ltd., Bromley, UK, 1993.

[3] V. V. Jikov, S. M. Koslov, and O. A. Oleinik, Homogenization of Differential Operators and Integral Functionals, Springer, Berlin, Germany, 1994.

[4] G. Nguetseng, "A general convergence result for a functional related to the theory of homogenization," SIAM Journal on Mathematical Analysis, vol. 20, no. 3, pp. 608-623, 1989.

[5] G. Allaire, "Homogenization and two-scale convergence," SIAM Journal on Mathematical Analysis, vol. 23, no. 6, pp. 1482-1518, 1992.

[6] L. Flodén, A. Holmbom, M. Olsson, and J. Persson, "Very weak multiscale convergence," Applied Mathematics Letters, vol. 23, no. 10, pp. 1170-1173, 2010.

[7] E. Zeidler, Nonlinear Functional Analysis and Its Applications IIA, Springer, New York, NY, USA, 1990.

[8] G. Nguetseng and J. L. Woukeng, " $\Sigma$-convergence of nonlinear parabolic operators," Nonlinear Analysis. Theory, Methods E Applications, vol. 66, no. 4, pp. 968-1004, 2007.

[9] A. Holmbom, "Homogenization of parabolic equations: an alternative approach and some correctortype results," Applications of Mathematics, vol. 42, no. 5, pp. 321-343, 1997. 


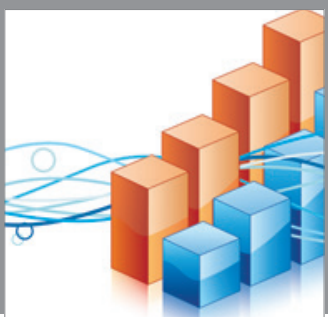

Advances in

Operations Research

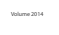

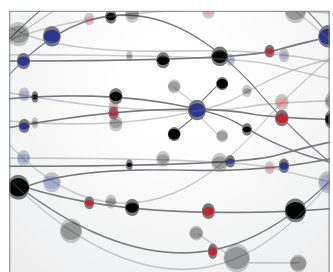

\section{The Scientific} World Journal
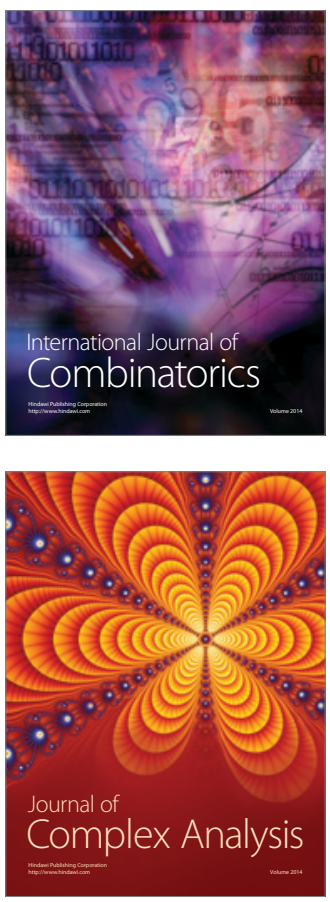

International Journal of

Mathematics and

Mathematical

Sciences
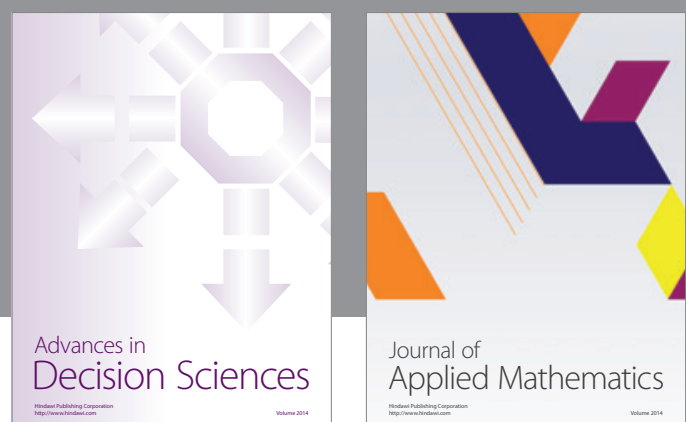

Journal of

Applied Mathematics
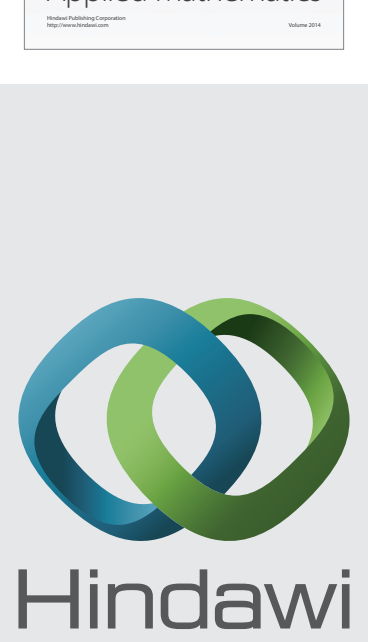

Submit your manuscripts at http://www.hindawi.com
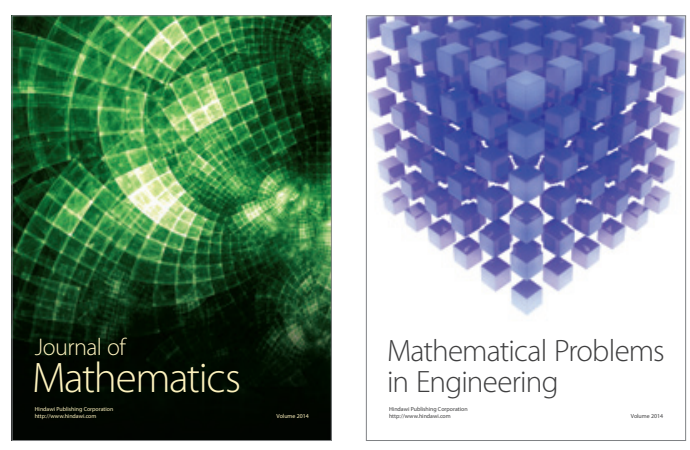

Mathematical Problems in Engineering
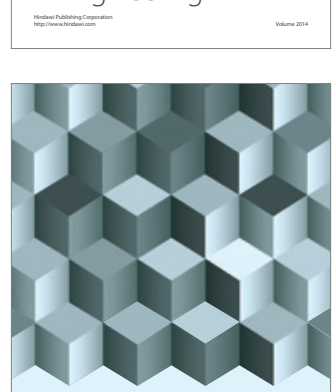

Journal of

Function Spaces
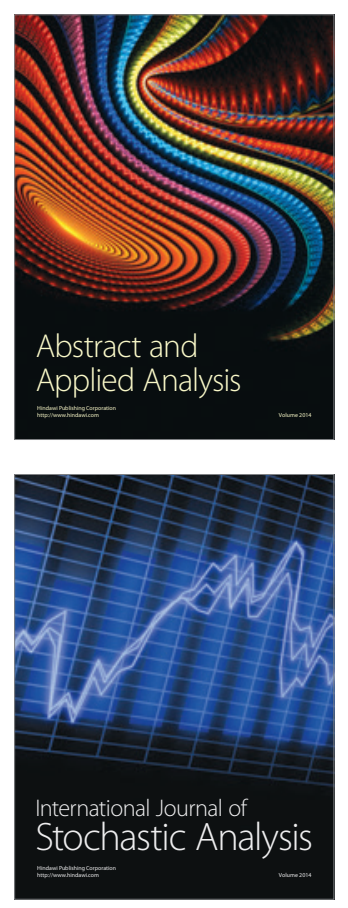

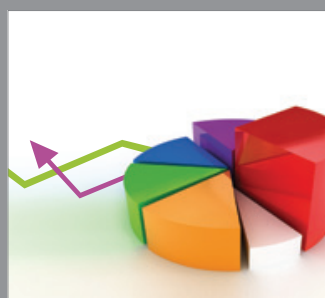

ournal of

Probability and Statistics

Promensencen
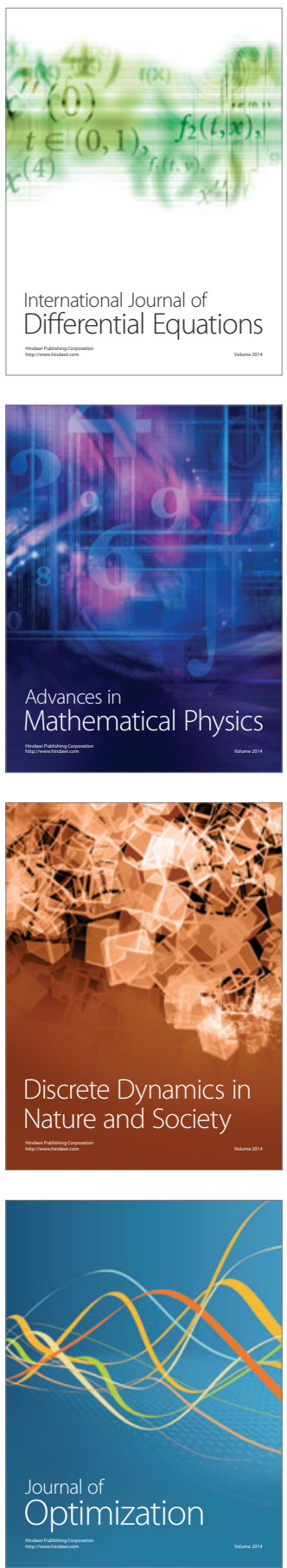